\title{
PROJETOS INDUSTRIAIS - BARREIRAS PARA A IMPLEMENTAÇÃO DA MODELAGEM DA INFORMAÇÃO DA CONSTRUÇÃO ${ }^{1}$
}

\author{
INDUSTRIAL PROJECTS - BARRIERS TO IMPLEMENTATION OF \\ BUILDING INFORMATION MODELING
}

\author{
Tássia Farssura Silva \\ tsa29v@hotmail.com \\ Karina Matias Coelho \\ Universidade de São Paulo (USP) \\ karina.matias@usp.br \\ Silvio Melhado \\ Universidade de São Paulo (USP) \\ silvio.melhado@usp.br
}

\begin{abstract}
Resumo
A introdução da Modelagem da Informação da Construção (BIM) ampliou a discussão sobre como melhorar a colaboração de modo a tirar proveito da nova tecnologia em projetos industriais; com uso do BIM, o aumento do número de interfaces e de tarefas relacionadas ao desenvolvimento do projeto exigem mudanças nas atuais práticas de gestão. Por meio de um estudo de caso referente à implementação do BIM em um projeto industrial, o objetivo do artigo é avaliar o impacto dessa implementação, destacando as principais dificuldades e pontos críticos resultantes da mudança tecnológica. Os métodos de pesquisa adotados foram: revisão da literatura quanto a gestão de projetos, tecnologia da informação na construção e outros conteúdos que fundamentaram a análise do estudo de caso. Por meio do contato com coordenador de projetos, engenheiros e líderes das disciplinas envolvidas, utilizando-se entrevistas semiestruturadas e análise de documentos, constataram-se deficiências do processo de projeto que impactaram o desenvolvimento das atividades e consequentemente, a qualidade do projeto estudado. Os fatores identificados foram divididos em grupos: pessoas; tecnologia; fatores de gestão. São apresentadas recomendações para evitar os problemas encontrados no caso estudado e alcançar os benefícios esperados com a implementação de BIM em projetos industriais.
\end{abstract}

Palavras-chave: BIM. Projeto colaborativo. Gestão de projetos. Projetos industriais.

\begin{abstract}
The introduction of Building Information Modelling (BIM) has raised a discussion on how to improve collaboration in order to take advantage of the new technology; with the implementation of BIM, the growth in the number of interfaces among players and tasks related to design detailing led to changes in the current management practices. Using a case study of an industrial project, the aim of this paper is to evaluate the BIM implementation impact in the sector, thus highlighting the main difficulties and critical points resulting from that technological change. The research methods adopted were literature review on design management and information
\end{abstract}

${ }^{1}$ SILVA, T.F.; COELHO, K.M.; MELHADO, S.. Projetos industriais - barreiras para a implementação da Modelagem da Informação da Construção. In: ENCONTRO BRASILEIRO DE TECNOLOGIA DE INFORMAÇÃO E COMUNICAÇÃO NA CONSTRUÇÃO, 7., 2015, Recife. Anais... Porto Alegre: ANTAC, 2015. 
technology and other subjects, in order to subsidise the case analysis. Some deficiencies in the design process have been found throughout project documents reviewing and interviews with the technical leaders, design coordinator and engineering designers and those deficiencies led to poor design quality. The paper groups the factors involved in three categories: people, technology and management. The results are presented as a set of specific recommendations to avoid the defeats found in the case study and reach the expected results and benefits of BIM implementation in industrial projects.

Keywords: Building information modelling. Collaborative design. Design management. Industrial projects.

\section{INTRODUÇÃO}

Um dos principais problemas enfrentados pela indústria da Construção Civil e, particularmente, pelo segmento de Edificações, é seu alto grau de fragmentação; uma indústria fragmentada é aquela em que nenhum de seus agentes exerce poder suficiente para influenciar o mercado sozinho (ZEGARRA et al., 1999). Além disso, no processo de produção de edificações, a fragmentação existe na divisão de responsabilidades entre os profissionais de projeto das diversas especialidades envolvidas, e entre profissionais, clientes e prestadores de serviços de projeto. Estas características estão na base cultural da competitividade entre os clientes, empreiteiros, subempreiteiros, fornecedores e todos os outros agentes, levando a um baixo grau de transparência e confiabilidade. Mesmo que o objetivo do empreendimento seja entregar um produto de qualidade, com um bom projeto e de acordo com as necessidades dos clientes, cumprindo com o prazo e custo (ELMANUELIM e GILDER, 2014), muitos modelos de gestão não são efetivamente orientados para isso. O processo de projeto de edifícios é dividido em quatro fases principais, que se referem às partes do processo do empreendimento: idealização do produto, projeto, construção e uso e operação. Estas fases são essencialmente sequenciais e conceitualmente progressivas; um maior nível de detalhe das soluções é adicionado a cada uma delas, ao mesmo tempo em que a possibilidade de escolher entre alternativas de projeto se reduz (MELHADO, 1994). A garantia de integração e coerência entre essas fases consecutivas é um dos maiores desafios no campo da gestão de projetos de construção.

Os problemas atuais envolvendo a gestão da qualidade do projeto e da execução das obras de construção são demandas nítidas por ações capazes de melhorar uma indústria que se mostra mais atrasada do qualquer outra, em sua tentativa de tornar-se mais competitiva e de prestar melhores serviços aos clientes e à sociedade como um todo (ARANDA-MENA et al., 2009). De acordo com esses autores, para reduzir conflitos, devem ser adotadas medidas para mitigar riscos e dotar a indústria de uma nova documentação da construção, por meio da tecnologia de modelagem de informações da construção (BIM). Portanto, nos anos recentes, a modelagem da informação da construção aparece como promotora da inovação a partir da integração dos agentes da construção e potencialmente pode melhorar os processos de trabalho nas quatro fases do projeto e nas interfaces entre eles. Espera-se que forneça colaboração eficiente, integridade de dados, documentação inteligente, acesso e recuperação de dados e projetos de alta qualidade, por meio de análises de desempenho melhorado, coordenação e planejamento multidisciplinar (GU e LONDON, 2010). No entanto, em um estudo realizado no Reino Unido, Eadie et al. (2013) demonstraram que o uso mais frequente de modelagem de informações acontece nas fases de concepção e de pré-construção (projeto detalhado). Na sequência, o uso mais comum foi na fase de construção em si e, finalmente, na fase de uso e operação. A mesma pesquisa também concluiu que a experiência de modelagem da informação da construção foi realizada dentro das equipes de projeto e poucos clientes são capazes de usar todo o seu potencial.

As edificações industriais mobilizam diversas especialidades na concepção e 
desenvolvimento do projeto desses empreendimentos $e$ as suas particularidades determinam as estratégias de ação de cada agente envolvido. Os principais agentes são: clientes, profissionais de projeto, consultores e empreiteiros, cada um com seus próprios objetivos e perspectivas particulares. Müller (2012) define a complexidade em projetos como um conceito multidimensional, função da confiança na capacidade de produzir os resultados esperados do projeto, da quantidade de informação a ser processada, da dinâmica e da incerteza dos projetos, além da interação entre os agentes envolvidos.

Projetos industriais apresentam uma característica muito própria. Dada a rápida evolução dos processos industriais, juntamente com a diversidade de produtos, cada novo projeto apresenta demandas do cliente e especificações que devem ser rapidamente compreendidas e atendidas. Por outro lado, além dessa dinâmica, o grupo profissional tradicionalmente envolvido em projetos industriais adota modelos de contratos que não estimulam comprometimento com os resultados finais e que levam a soluções pouco integradas, reduzindo assim o potencial de integração oferecido pelo uso do BIM (SILVA e MELHADO, 2014).

A partir da análise de um estudo de caso, o objetivo deste trabalho é avaliar o impacto do BIM no setor de projetos industriais, destacando as principais dificuldades e os pontos críticos resultantes dessa mudança tecnológica. Os resultados da avaliação acima são apresentados como um conjunto de recomendações para atingir os resultados esperados e benefícios do BIM em casos semelhantes, baseados nas melhores práticas de gestão de projetos.

Os métodos de pesquisa adotados foram: revisão da literatura sobre gestão de empreendimentos e gestão de projetos, sobre modelagem de informações da construção e outros conteúdos que fundamentam o trabalho, além da realização do estudo de caso. Um dos critérios para selecionar a empresa estudada foi obter informação prévia sobre a organização; considerou-se, por exemplo, se a empresa teria pelo menos um projeto desenvolvido em BIM e a disponibilidade para fornecer as informações solicitadas pela pesquisa, bem como o acesso aos profissionais envolvidos para proceder às entrevistas. Uma vez que a empresa do estudo de caso foi selecionada, as informações necessárias foram obtidas com o profissional responsável pela coordenação do projeto, engenheiros e líderes das disciplinas envolvidas, incluindo entrevistas técnicas e análise de documentos como contratos, projetos e atas de reunião. O estudo começou a ser desenvolvido em 2011 quando a informação principal foi coletada. Depois disso, os detalhes e o estudo documental em profundidade foram realizados no final de 2013. Um questionário semiestruturado foi desenvolvido como base para realizar as entrevistas.

\section{BIM - MODELAGEM DA INFORMAÇÃO DA CONSTRUÇÃO}

A escolha de modelagem da informação da construção como uma alternativa aos modelos de representação do projeto justifica-se pela sua abordagem sistêmica e integrada de todas as fases do ciclo de vida, reunindo-as em um modelo único e compartilhado de informações, levando a melhores resultados e menos interferências com a produção e entre as informações usadas pelas várias partes envolvidas no processo (ABAURRE, 2013).

Segundo Underwood e Isikdag (2011), embora o principal papel do BIM seja facilitar a fase de projeto de um empreendimento de construção, a modelagem da informação da construção pode ter uma ampla gama de funções, tais como a ligação entre edificações e espaços urbanos exteriores, facilitando a partilha de informações entre as diversas partes interessadas e os aplicativos de software que as utilizam, permitindo a construção, utilização e gestão compartilhada dessas informações, facilitando a simulação de processos de 
construção e apoiando as operações de resposta a emergências.

Por ser um banco de dados único para o desenvolvimento do projeto, a tecnologia BIM permite fazer alterações dinâmicas na modelagem que se refletem de forma positiva, uma vez que podem ser vistas em tempo real por todas as áreas técnicas envolvidas. Dentre vários benefícios e vantagens oferecidas pelo BIM, alguns autores destacam: aumento da produtividade, melhoria da qualidade gráfica de apresentação do projeto, melhoria da comunicação interdisciplinar, eliminação da redundância de dados, redução do retrabalho e de erros, entre outros. No entanto, a estratégia da implementação de BIM tem um longo caminho a percorrer para atingir a maturidade e precisa ser continuamente melhorada (UNDERWOOD e ISIKDAG, 2011).

O uso do BIM em empresas projetistas pode beneficiar o processo de projeto. No entanto, a cultura das empresas no setor, o fluxo informal de informações e de documentos entre as partes envolvidas, o uso inadequado das ferramentas e a falta de formação dos projetistas para as novas ferramentas afetam o processo de projeto e, consequentemente, a qualidade do projeto (ITO, 2007). O uso do BIM requer uma nova maneira de pensar. Habituados ao desenvolvimento de projetos usando metodologias tradicionais e processos de representação em duas dimensões (2D), os profissionais de projeto se veem diante da necessidade de mudar seus conceitos, ao usar o BIM. $E$ as equipes que eram tradicionalmente fragmentadas, devem se tornar uma única equipe, integrada e colaborativa.

Khosrowshahi e Arayici (2012) descrevem a estrutura de níveis de maturidade do BIM em estágios. Esses estágios de maturidade, que são brevemente descritos abaixo, são referências úteis para classificação sistemática da implementação do BIM.

Pré-BIM - refere-se à prática do processo de concepção tradicional composto por ineficiências e barreiras significativas. Um grande volume de informações de projeto é comunicado por meio de documentos em papel (desenhos e documentos escritos), que podem ser facilmente perdidos ou danificados. Uma gestão pobre de informações leva à compreensão incompleta das soluções de projeto, a ineficiência funcional, conflitos entre elementos de projeto e trabalho impreciso.

BIM Estágio 1 - refere-se à migração de 2D para 3D e modelagem. A documentação passa a ser baseada em objetos digitais. O modelo BIM é feito de elementos reais, que são corretamente representados sob todos os pontos de vista. O modelo BIM ainda é desenvolvido sob disciplinas individuais e os produtos finais são principalmente arquivos CAD.

BIM Estágio 2 - evolui da modelagem individual para a colaboração e a interoperabilidade. Gestão e concepção de edifício são processos altamente complexos que exigem a evolução em comunicação e colaboração entre todos os membros da equipe de projeto. A maturidade da fase 2 requer comunicação integrada e o compartilhamento entre as partes interessadas, para apoiar esta abordagem colaborativa dos dados criados em projeto.

BIM Estágio 3 - transição de colaboração para a integração e reflete a verdadeira filosofia do BIM. O modelo da fase 3 torna-se interdisciplinar, permitindo criar modelos completos desde a fase inicial do projeto. No estágio 3 , o conteúdo do modelo vai além de informações de objetos semânticos, incluindo assim a inteligência de negócios e os métodos construtivos.

Mesmo que cada continente, região ou país esteja hoje em diferentes etapas do uso do BIM, pode-se adotar os estágios acima como uma referência universal. A implementação da modelagem da informação da construção, em uma perspectiva de longo prazo, vai mudar não só as práticas profissionais, mas também as relações contratuais entre as partes (MANZIONE, 2013). Esse autor afirma que, graças à tecnologia envolvida na construção do modelo BIM, o trabalho colaborativo com várias partes é possível; no entanto, para chegar a 


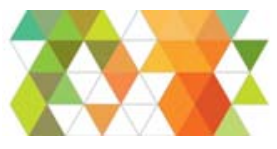

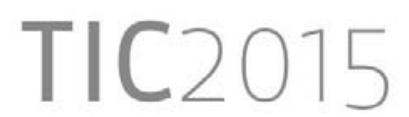

um estágio de maturidade mais avançado, além da adoção das novas práticas, serão necessárias mudanças nas relações contratuais, criando novos objetivos para os projetistas.

Uma utilização eficaz do BIM requer inúmeras mudanças, tais como: alterações em contratos, escopos e entregas; mudanças no ambiente de trabalho, buscando integração multidisciplinar e foco no desempenho da equipe como um todo e não como disciplinas individuais; alterar o processo de comunicação do projeto; entre outros. Estas mudanças podem gerar resistência dos profissionais, principalmente dos mais experientes, o que pode contribuir para a dificuldade na implementação do BIM em uma organização.

De acordo com Jensen e Jóhannesson (2013), o mais importante no processo de implementação parece estar em realizar a transformação em pequenos passos. O papel da gestão de recursos humanos para implementação de BIM pode ser crítico. É natural que alguns profissionais sintam que eles estão ameaçados pelas mudanças. Isto só pode ser visto como uma boa razão para as empresas implementarem BIM de forma gradual. Os resultados da pesquisa realizada pelos autores mostram a importância de envolver a alta direção na implementação do BIM, porque a liderança deve ser convencida dos benefícios do BIM e ele deve ser um elemento central da estratégia de negócios, não apenas mais um sistema de software. Caso contrário, será difícil obter recursos suficientes para o processo de transformação. Se uma empresa deseja implementar o BIM no processo de concepção e projeto, significa que mudanças fundamentais devem ser feitas em todos os seus processos. O período de transição, quando se altera de métodos tradicionais de trabalho para processos BIM é um momento crítico para todas as empresas e tem que ser considerados os interesses e o contexto de cada empresa. É necessário difundir o conhecimento para todos os colaboradores da empresa a fim de torná-los cientes dos potenciais destes novos processos e atividades. Uma plena aceitação, com a participação e a exploração das possibilidades não ocorrerá até que todos estejam convencidos das vantagens da mudança.

\section{ESTUDO DE CASO}

\subsection{Realização do estudo de caso}

O estudo de caso envolve um empreendimento industrial, projetado por uma empresa de engenharia que tem sua sede na cidade de São Paulo. No caso estudado, foram identificadas algumas deficiências na utilização de modelagem da informação da construção no processo de projeto, deficiências essas que impactaram o desenvolvimento das atividades e, consequentemente, a qualidade do projeto.

O estudo de caso compreendeu as seguintes etapas: definição da empresa e projeto a serem investigados; proposta dos protocolos de pesquisa para o estudo de caso; agendamento e realização das entrevistas técnicas; compilação das informações obtidas.

\subsection{A empresa estudada}

A empresa de engenharia analisada neste estudo é uma empresa brasileira, que trata da concepção, gestão, engenharia e fornecimento de serviços EPC / EPCM. Tem uma experiência de 25 anos no mercado brasileiro, atuando por meio de sua sede principal em São Paulo, mas também com escritórios em outras cidades brasileiras, além de alguns grupos-tarefa estabelecidos em outras sedes, no Brasil e no exterior, em países da América do Sul e América Central, Europa, África e Ásia. A empresa tem uma equipe de engenharia com cerca de 2.300 profissionais (83\% trabalham em atividades técnicas e $17 \%$ em atividades administrativas), com clientes em diferentes setores como mineração, metalurgia, 
aço, fertilizantes, óleo \& gás, petroquímica, infraestrutura, energia, construção de docas, gerenciamento de construção e implementação de sites. A equipe de gestão da empresa, apoiada pelos engenheiros de projeto e pelo departamento de planejamento, executa a gestão dos seus projetos. Quatro diretorias de setores industriais e de gestão supervisionam diretamente os coordenadores de projetos e planejadores.

\subsection{O projeto estudado}

O projeto envolve o desenvolvimento do projeto detalhado de ampliação de uma fábrica composta por três unidades de produção de cosméticos, fragrâncias e higiene pessoal. $\mathrm{O}$ escopo do projeto contratado inclui o desenvolvimento de estudos sobre novos edifícios e extensões do prédio existente, áreas de produção, enchimento, embalagem e utilidades. Uma equipe de projeto foi designada para desenvolver todo o escopo do projeto, a partir de um projeto conceitual consolidado pelo cliente, entregando os documentos (desenhos, memoriais, planilhas quantitativas) sob a forma de projeto básico e detalhado dos edifícios e áreas do site.

Uma das principais solicitações do cliente foi que todo o projeto deveria ser desenvolvido com a tecnologia de modelagem da informação da construção (BIM), desde o início.

\subsection{Processo de projeto usando a "plataforma BIM"}

No caso estudado, o estágio de maturidade no uso de BIM pode ser considerado no primeiro nível de implementação (Estágio 1). Mesmo considerando o que se espera do Estágio 1, algumas deficiências no processo de projeto com o uso da "plataforma BIM" impactaram o desenvolvimento de atividades e, consequentemente, a qualidade do projeto:

- Inexperiência no desenvolvimento de modelos tridimensionais;

- Deficiência no esclarecimento do escopo do projeto detalhado, desde as reuniões iniciais (KOM);

- Falta de definição do nível de desenvolvimento (ND ou LOD) e demais requisitos para o modelo BIM.

Com base em declarações dos funcionários da empresa de projeto, as dificuldades que foram identificadas estariam ligadas aos seguintes fatores:

a) Falta de tempo e planejamento para a implantação da tecnologia - estas eram as principais dificuldades apontadas. No caso do projeto estudado, ainda havia um fator agravante, pois não foi contratado consultor técnico para a implementação, sendo que a cultura BIM não existia na empresa;

b) Falta de profissionais qualificados - havia poucos profissionais com experiência na modelagem da informação da construção (BIM), o que levou a empresa a fornecer treinamento, mas apenas para um grupo específico de empregados;

c) Resistência a mudanças pela equipe - na empresa estudada, havia uma resistência mais elevada dos funcionários mais experientes;

d) Falta de infraestrutura de $\mathrm{TI}$ - o novo software utilizado pela empresa exige computadores com um poder de processamento maior do que aquele que anteriormente era usado somente para CAD. A mudança de equipamento era necessária para ter um melhor desempenho, mas a falta de equipamentos disponíveis na empresa gerou atrasos no desenvolvimento das atividades planejadas;

e) deficiências próprias do software (ou desconhecimento da forma correta de operá-lo) - 
ineficiências e problemas quanto a processar o modelo de fabricação de escadas com dois níveis foram detectadas, como mostrado nas Figuras 1 e 2. Na Figura 1, observa-se que foram detectados elementos adicionais no modelo e um equívoco no posicionamento da escada com dois patamares. Já na Figura 2, foi observada a falta de elementos no modelo e outro equívoco no posicionamento do mesmo tipo de escada.

f) Compatibilidade - por uma questão política da empresa, a projetista usou software de fornecedores diferentes para as equipes envolvidas no desenvolvimento do projeto (ver organograma da equipe na Figura 3). Por exemplo, a modelagem de arquitetura utilizava REVIT, a engenharia mecânica usava Solid Works, a engenharia de instalações usava AutoCAD 3D e outras disciplinas simplesmente foram representadas em 2D. Apesar da possibilidade do uso de arquivos IFC para comunicação entre as diferentes plataformas, problemas relativos à compatibilidade entre softwares foram detectados, como pode ser visto na Figura 4, na qual os tubos são representados por linhas, não por sólidos. A compatibilização entre as disciplinas é comprometida, dado que a tubulação não é representada no modelo com suas reais dimensões.

g) Adaptação às normas brasileiras de construção - a equipe de arquitetura relatou que grande parte da biblioteca existente no software é estrangeira e uma adaptação a normas nacionais é imprescindível (necessária qualificação de profissionais para edição e desenvolvimento de objetos virtuais e suas famílias).

h) Tamanho dos arquivos - os arquivos de modelos gerados são muito maiores que os de CAD, gerando mais dificuldades, devido aos aspectos descritos no item (e).

i) Gestão do projeto - falhas ou falta de gestão e planejamento envolvendo as alterações necessárias no processo de projeto também foram observadas.

Figura 1 - Problemas detectados na modelagem de escadas com dois níveis - erro de modelagem mostrando elementos adicionais

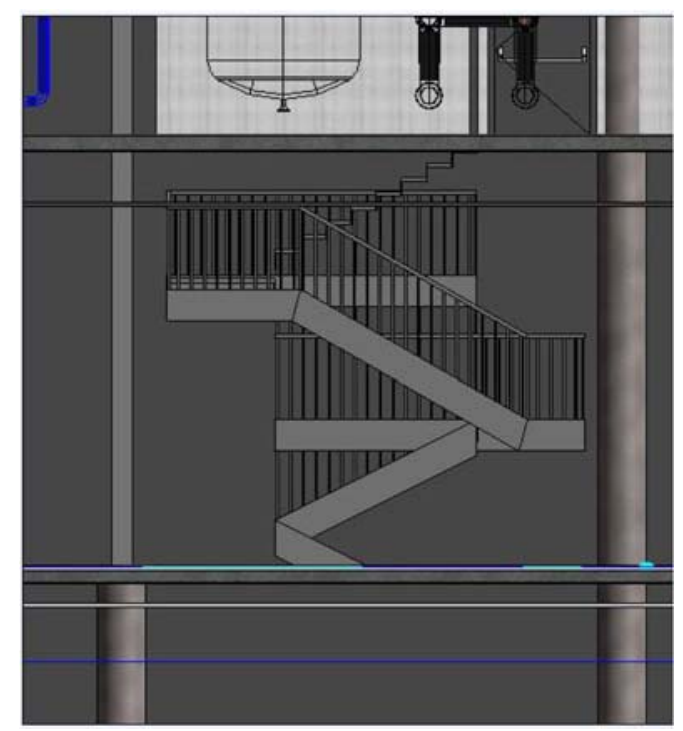

Fonte: dados do estudo de caso 


\section{TIC2015}

Figura 2: Problemas detectados na modelagem de escadas com dois níveis elementos faltando no modelo

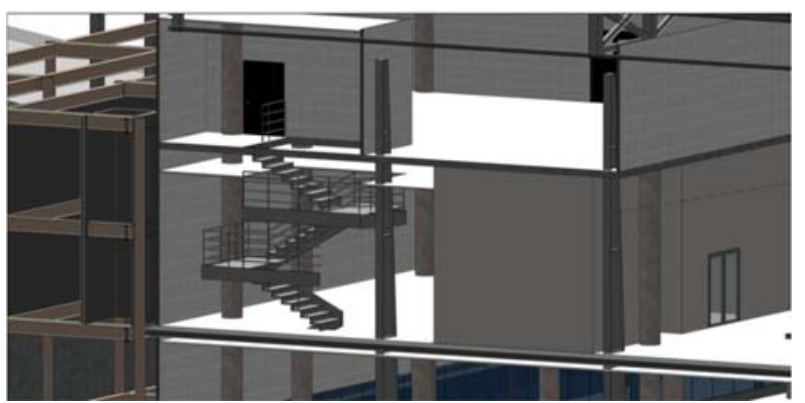

Fonte: dados do estudo de caso

Figura 3: Organograma do projeto

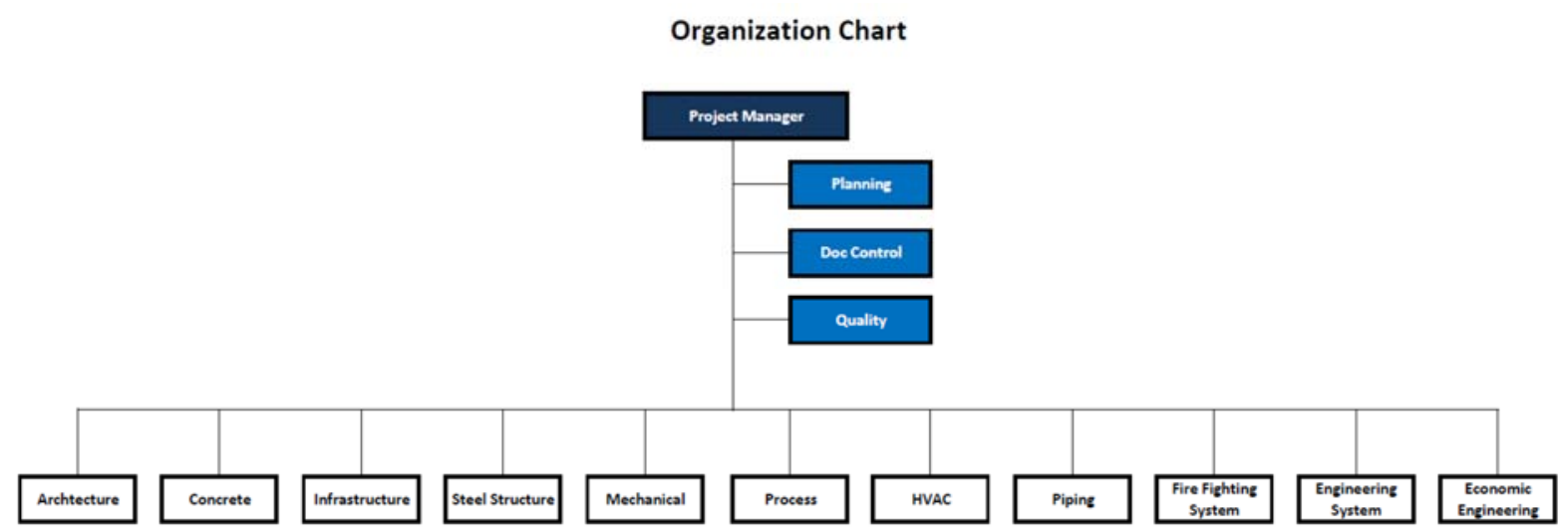

Fonte: dados do estudo de caso

Figura 4: Problemas de compatibilidade

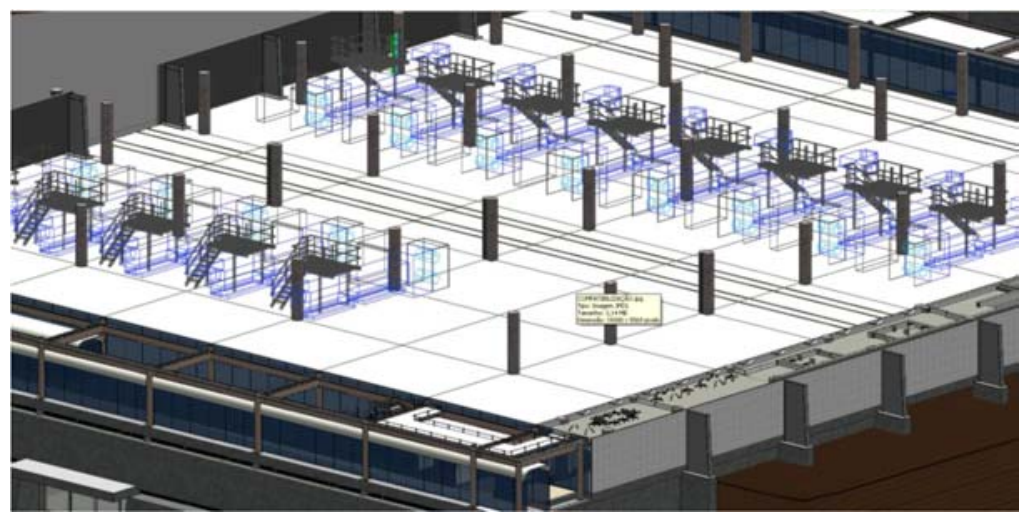

Fonte: dados do estudo de caso

\section{CONCLUSÃO}

As dificuldades identificadas no estudo de caso (seção 3.4) podem ser divididas em três 
grupos principais de fatores, de acordo com sua natureza: pessoas, tecnologia e fatores de gestão, que são individualmente comentados abaixo, bem como apresentadas as consequentes recomendações.

Pessoas: dificuldades descritas nos itens a, b e c. No contexto brasileiro, para a maioria dos projetos de construção, a primeira experiência usando BIM tem sido orientada por uma empresa de consultoria contratada que ajuda não só no software e treinamento do sistema, mas também cria um novo processo de trabalho em equipe, propõe uma revisão do processo de projeto e assim por diante. Recomenda-se que trabalhar com BIM requer não só aprender as ferramentas necessárias, mas também uma nova maneira de pensar e um novo processo de projeto.

Tecnologia: (d), (e), (f) e (g). Ao implementar o BIM, os requisitos de hardware e software exigem uma quantidade significativa de investimento, que deve ser suportada pelo orçamento do projeto em si ou pela empresa de projeto, como uma iniciativa geral para garantir as condições de modelagem de todos os projetos contratados; recomenda-se que o investimento seja realizado não só em hardware, software e largura de banda, mas também na seleção de software apropriado, observando-se as questões de interoperabilidade. Dessa forma, o investimento em tecnologia pode ser direcionado para solucionar problemas encontrados no caso estudado, tais como compatibilização, deficiências da modelagem e atrasos e permitir melhoria da qualidade gráfica de apresentação do projeto, melhoria da comunicação interdisciplinar, eliminação da redundância de dados, redução do retrabalho e de erros, entre outros.

Gestão: (h) desde a apresentação da proposta, ou seja, mesmo antes do início do projeto, recomenda-se considerar a necessidade de uma biblioteca de elementos de projeto, que pode ser fornecida por um especialista contratado para o projeto específico, uma empresa de consultoria BIM ou selecionando um escritório de projeto que já tem a biblioteca necessária.

i) trabalhar com BIM requer mudanças na cultura de processo de projeto, mudanças em que planejamento e de gestão são essenciais para uma implementação bem-sucedida; e essas mudanças, por conseguinte, exigem preparação e capacitação prévios.

Além disso, no estudo de caso, várias outras questões apontadas por outros autores foram igualmente encontradas. O caráter fragmentado da indústria da construção e a falta de colaboração entre os agentes, bem como a necessidade de qualificação dos profissionais de projeto e novos modelos de contrato produziram claras consequências. As expectativas dos clientes não foram satisfeitas e a modelagem de informação da construção não contribuiu para alcançar soluções de projeto melhores e mais integradas.

Como exemplo de pontos-chaves para alcançar níveis mais elevados de maturidade no uso de BIM, novos modelos de contrato devem ser adotados, orientados à colaboração, agregando valor ao esforço para evitar conflitos e erros de modelagem. Além disso, métodos aperfeiçoados de gestão do projeto, adotando KPIs (MANZIONE, 2013) são também uma questão estratégica para melhorar o nível de maturidade nos projetos industriais.

Diante dos problemas encontrados e lições aprendidas naquele primeiro projeto, a empresa estudada implantou algumas melhorias, porém estas não foram suficientes para garantir os benefícios oferecidos pelo BIM. Atualmente, a maior parte dos projetos é desenvolvida em modelagem; mas, como não há uma nova cultura do processo de projeto, assim como um novo sistema de gestão, ainda não é possível produzir projetos para atender integralmente às expectativas dos clientes e os objetivos da organização quanto ao uso de BIM.

Em conclusão, como em qualquer outro segmento da construção, os projetos industriais 


\section{TIC2015}

podem realmente se beneficiar das novas ferramentas de tecnologia da informação, se devidamente implementadas. No entanto, sem planejamento para implantação de uma inovação tecnológica e organizacional, alterações no processo de projeto e uma ação de qualificação de profissionais eficiente, anterior à introdução de técnicas de modelagem, os resultados esperados tendem a não serem alcançados.

\section{REFERÊNCIAS}

ABAURRE, M.W. Modelos de contrato colaborativo e projeto integrado para modelagem da informação da construção. Dissertação de Mestrado, Escola Politécnica da Universidade de São Paulo, 2013.

ARANDA-MENA, G. et al. Building information modelling demystified: does it make business sense to adopt BIM? International Journal of Managing Projects in Business, Bingley, v.2, n.3:.419$34,2009$.

EADIE, R. et al. BIM Implementation throughout the UK construction project lifecycle: An analysis. Automation in Construction. Vol. 36: 145-151, 2013.

ELMANUELIM, A.; GILDER, J. BIM: innovation in design management, influence and challenges of implementation. Architectural Engineering and Design Management, v.10 n.3-4: 183-99, 2014.

GU, N.; LONDON, K. Understanding and facilitating BIM adoption in the AEC industry. Automation in Construction, v. 19, p. 134-44, 2010.

ITO, A.L.Y. Gestão da informação no processo de projeto de arquitetura: estudo de caso, Universidade Federal do Paraná, Curitiba, 2007.

JENSE, P.A; JÓHANNESSON, E.I. Building information modelling in Denmark and Iceland. Engineering, Construction and Architectural Management, Bingley, v.20, n.1: 99-110, 2013.

MANZIONE, L. Proposição de uma estrutura conceitual de gestão do processo de projeto colaborativo com o uso do BIM. Tese (Doutorado), Escola Politécnica da Universidade de São Paulo, 2013.

KHOSROWSHAHI, F.; ARAYICI, Y. Roadmap for implementation of BIM in the UK construction industry. Engineering, Construction and Architectural Management, Bingley, v.19, n. 6: 610-35, 2012.

MELHADO, S.B. Qualidade do projeto na construção de edifícios: aplicação ao caso das empresas de incorporação e construção. Tese (Doutorado), Escola Politécnica da Universidade de São Paulo, 1994.

MÜLLER, R, et al. Relationships between leadership and succes in different types os project complexities. IEEE Transactions on Engineering Management, v. 59, $n^{\circ}$ 1, 2012.

SILVA, T.F.; MELHADO, S. Gestão de Projetos Industriais. São Paulo, PINI, 2014.

UNDERWOOD, J.; ISIKDAG, U. Emerging Technologies for BIM 2.0. Construction Innovation, Bingley, v.11, n. 3, p. 252-258, 2011.

ZEGARRA, S.L.V. et al. A tecnologia da informação e a industria da construção de edificios. In: RESENDE, M.F. et al. (eds.). Simpósio Brasileiro de Gestão e Qualidade e Organização do Trabalho: A competitividade da Construção Civil no Novo Milênio. Recife, UPE-ANTAC, 1999. 